\title{
Coping With Stress the Bach Flower Way
}

\author{
Dr Balaji Deekshitulu P V*
}

\section{ABSTRACT}

In this review article the author argues that the complete presentation is relaxation of stress through neither for Possible of Bach flower remedies as inevitable part of physical and mental health for stress busted leg.

Keywords: Stress, Bach flower Remedies.

\section{INTRODUCTION}

Bach flower remedies used to treat stress can the best of emotional healing. Dr. Edward Bach who was an English physician found out the importance of the essence of flowers in the healing process way back in the 1930s. It has been noted that the Egyptians used flower essence. This was noted and developed in to Australian Bush Flower Essences which have 66 remedies while the Bach Flowers Essences have a total of 38 remedies by Ian White.

These are some of the Bach flower remedies used to treat stress that work the best. Aspen is the leaf that has an extremely delicate nature and the slightest of breeze will make it shiver and quake. If you have an anxiety because of no particular reason then this is the flower remedy that will help you by instilling a deep rooted feeling of security and boost confidence levels.

Stress management strategies such as Bach Flower Remedies are an excellent way to begin the long, hard climb back from the chaotic, stressful world, in which many of us reside. For emotional stress management, Bach Flower Essences are, by far, the best of the stress management products on the market today. The amazing thing is that this winner in the stress management strategies realm is not even new. It was first discovered in the 1920's and 1930's by a medical doctor in England named Edward Bach.

\section{Mind-body connections}

Physical and mental symptoms are often linked, for several reasons. Emotional changes can be a result of physical illness and its treatment, developing in response to factors such as the following:

\footnotetext{
*Guest Lecturer in Psychology and Psychologist \& Alternative Medicine Practitioner, Sri Balaji Clinic Tirupati , India

(C) 2014 B Deekshitulu; licensee IJIP. This is an Open Access Research distributed under the terms of the Creative Commons Attribution License (http://creativecommons.org/licenses/by/2.0), which permits unrestricted use, distribution, and reproduction in any Medium, provided the original work is properly cited.
} 
- Distressing symptoms such as pain, nausea or breathlessness

- Restriction of activity due to weakness or immobility

- Concern about the future medical outcome

- Concern about the wider impact, for example on family and finances

- Direct biological effects on the structure or function of the brain.

Imbalances of mood or personality, and the experience of stress, are also implicated in the causation of physical illness. Broadly speaking, this can happen via two pathways:

- The direct influence of thoughts and emotions on bodily systems, especially the endocrine (hormonal) and immune systems

- The increased likelihood of unhealthy lifestyle practices, such as smoking or excessive drinking and self-neglect, among people who are unhappy or stressed.

Many holistic healers believe that psychological imbalance is the root cause of most disease. However it is not the only cause and material factors such as genetic predisposition, infections, poor nutrition and toxins in the environment usually play an important part as well.

Therefore, whereas no disease should be considered 'all in the mind', most diseases do have some 'psychosomatic' component. The role of the Bach flowers is to balance the mind-body relationship, and thereby improve both the mental adjustment to an illness and the capacity for physical self-healing.

Chancellor (1971) Bach's therapy is a modification of Aromatherapy, with the exclusive use of flowers. It is believed that flower essences define moods and emotions. Personality disturbances, loneliness, fear, indecision, despondence, despair, over care, over sensitivity, insufficient interest in self and others, etc., are among the 38 states of the disturbed mind identified by Bach for which he prescribed flowers of 38 different species

Jane Evans (1974) asked that detailing the benefits of the Bach Flower Remedies, flowerbased alternative treatments used for depression, anxiety, insomnia, and stress.

Jeffrey R. Cram, Ph.D. (1997) asked that psychophysiology provides a viable tool by which to investigate subtle energies, especially when guided by the metaphysical wisdom of the ages. This represents a blending of the old and new, the East and West. For what is the basis of science but to describe what is all ready known, using the latest tools of our culture. That the Five-Flower Formula has been demonstrated to significantly attenuate the stress response lends credence to the use of these substances in the treatment of stress-related disorders. 
David Lord (1999) found that the Bach flower remedies easily healing become part of illnessprevention routines and relaxation of mind.

Element Books, Incorporated (1999) asked that various Bach flower remedies are best stress busters. We are explain rescue remedy, white chestnut etc..

Christine Zaza and Natalie Baine (2002) significantly associated with more intense pain. Based on several criteria, the evidence is considered Strong for psychological distress, Moderate for social support, and Inconclusive for coping. This review suggests that comprehensive chronic pain assessment should include routine screening for psychological distress.

Scott Kessman (2005) says that Bach Flower Remedies for Relieving Stress and Other Negative Emotions in People and Animals.

Robert Halberstein et al. (2007) significant that difference between pretest and posttest scores. The results suggest that Bach Original Flower Essence (BFE) Rescue Remedy may be effective in reducing high levels of situational anxiety.

Estella Ritter (2007) asked that the comprehensive help for stress related anxiety and anxiety disorders. Anxiety being the main mental health concern for women, and second only to substance abuse in men, The Treatment of Anxiety \& Panic with Bach Flower Remedies offers both a practical and comprehensive guide to everyone struggling with panic attacks, anxiety attacks, social fears, generalized anxiety, or obsessive compulsive behaviors. Representing a fluid, self-directed process to emotional healing the goal of the technique is to present a very contemporary, user-friendly, and immediate response to emotional distress. Working by specifically targeting every type of negative state of mind reveals an amazing, subtle energy approach that is neither created in the language of pop culture, nor new age therapy. By explaining step-by-step how to use one of the most effective healing tools nature has provided to us, this book in a non-clinical voice, addresses the debilitating impact of anxiety, and shows how to effectively overcome anxiety driven symptoms in order to achieve a lasting recovery. Rather than just covering up and continuing to suppress emotional difficulties with drugs, this book supplies the tools needed to manage anxiety, and after that to resolve emotional problems The information can be used to develop one's own self-help program, or, as an adjunct to other healing modalities, the technique will reliably deliver clarity and a lifetime of emotional assistance.

Cornelia Richardson-Boedler(2007)asked that these remedies to totality of mental/ emotional and physical symptoms.

Judy horward(2007)significance of the therapeutic value of these remedies in relation to pain above that of a placebo, the results are encouraging. In particular, relief of negative emotions and promotion of positive thought including how clients opened up about, and dealt with, emotional issues. The indication is that potential for Bach flower remedies as a therapeutic agent in the 
relief of pain does exist and is worthy of further qualitative and quantitative investigation through robust, purpose-designed studies to replicate and progress the results shown here.

Kylie Thaler (2009) asked that Most of the available evidence regarding the efficacy and safety of BFRs (Bach flower remedies) has a high risk of bias. We conclude that, based on the reported adverse events in these six trials, BFRs are probably safe. Few controlled prospective trials of BFRs for psychological problems and pain exist. Our analysis of the four controlled trials of BFRs for examination anxiety and ADHD indicates that there is no evidence of benefit compared with a placebo intervention.

Vinton McCabe (2009) asked that Bach's remedies and their myriad uses. More important, he gives a colorful character portrait for each of the remedies; portraits created with insight, humor, and an understanding of human emotions and behaviors of stress.

Jennifer Barraclough (2012) asked that Bach remedies to help with some of the psychological issues which may arise in association with physical illness. It is not just about resolving the 'negatives' such as anxiety stress and sadness, but also includes promoting the 'positives' such as enhanced creativity and more loving relationships.

\section{STRESS MANAGEMENT}

Too much 'stress' can have negative effects on mind, body and behavior, and it is generally accepted that many, perhaps most, forms of both mental and physical illness are stress-related to some extent. However, the term is often used very loosely. One broad definition reads as follows: The state of physical or emotional tension which results when the demands facing a person challenge their ability to cope.

This definition implies an imbalance between external pressures and internal resources.It is not only major adverse events and difficulties such as bereavement, divorce, poverty and unemployment, natural disasters and wars which lead to stress. Apparently minor hassles of life, such as having to work with an irritating colleague or live in a noisy environment or cope with too many emails, can be stressful too. In general, the situations which cause the most stress are those which we perceive as being imposed against our will, and as beyond our control. And even 'pleasant' events such as going on holiday can be stressful if they involve too much stimulation and change.

Perceptions of stress vary enormously depending on attitudes, beliefs and personality. The same situation can be an exciting challenge for one person, but a terrifying ordeal for another. Many people who are stricken by some major adversity find themselves coping much better than they would have expected. One of the best 'buffers' against stress of any kind is having a strong network of relationships with people you can confide in, and rely upon for support. 
People also vary enormously according to how many stressful situations they experience in their lives. Some seem to attract them by their own attitudes and behavior and, of course, one stressful event frequently leads on to a cascade of others.

Early manifestations of being under too much stress include feeling anxious, irritable, overwhelmed, eating or sleeping too much or too little, tiredness, digestive upsets and various aches and pains. These are reversible symptoms which can be viewed as warning signals. If they are ignored, and the root problem is not removed, more serious mental or physical conditions may ensue. The physiology and biochemistry involved has been extensively studied, one marker of stress being over-secretion of the hormone cortisol.

Having a physical illness is in itself a source of mental stress, so there is often a vicious circle operating - not only does stress contribute to ill-health, but ill-health creates further stress. It is therefore often impossible to tell which came first, but in practical terms it probably does not really matter too much. The important thing is to accept that stress and negative states of mind, whatever their cause, tend to have adverse effects on medical outcomes and quality of life, and therefore it is always worthwhile to try to turn them around into more positive ones. How you are feeling now is usually the best starting-point, and this may also provide valuable pointers to the long-term issues which have been important throughout your life.

Stress cannot be avoided entirely, but ways of minimizing its impact include: changing the external circumstances if possible, changing your own perceptions of these circumstances, and practicing the art of acceptance.

Many other parts of this book are relevant to stress management: see for example the sections on Attitudes, Boundaries, Relationships, and Relaxation. And while all 38 of the Bach flower remedies are concerned with reducing stress in one way or another, two of them merit special mention here. Elm is for people who, though basically very capable, feel overburdened with responsibilities or overwhelmed with pressures and doubt their ability to cope. Walnut helps to protect from outside influences and change.

Bach Flower Remedies to Treat Stress can be used in the form of Agrimony. This is also referred to as Church Steeples. It is an herb that is found all over England and grows in abundance along the hedges and farmlands. It is extremely valuable to those who have great deal of anxiety and need to put on a brave face for the world. This constant making of an effort makes it really difficult for them to put on pretence and to hide this they overindulgence in food.

Cherry Plum is the best Bach Flower Remedy for Treat the Stress. It is best for people who are worried about losing their control and therefore feel edgy all the time. This is the most wonderful of options for people who are high strung and have sudden bursts of anger which is linked with anxiety. This can also be used to treat screaming fits in children. Red Chestnut is the perfect thing for people who are anxious about those around them. They tend to be over 
protective and alert all the time when around friends and family. They also tend to fear the worst and worry themselves and fritter away gainful energy on it.

Rock Rose is an extremely good example of Bach Flower Remedies to Treat Stress attacks linked with terror and panic. It is very good in bringing on a calmness and fortitude to the person. This is specifically in the case of people who have been in an accident or a natural disaster. These are really the best of flowers that can heal.

Bach flower remedies promote relaxation by dissolving mental tension. Examples are Vervain for over-enthusiasm, Oak for an over-developed sense of duty, Rock Water for excessively high ideals and self-discipline, Beech and Vine for tendencies to criticize and control, and White Chestnut for repeated worrying thoughts

Mimulus is a yellow colored trumpet like flower that is used to address stress \& fears and anxiety that occur due to some unknown factors. These could be in children and adults who are shy and sensitive. This is a great treatment for people with anxiety that is linked with meeting new people and going out.

\section{CONCLUSION}

Most of those who take Bach flowers soon report positive effects such as feeling calmer, lighter and more in control. These remedies are extremely safe. Stress cause problems for anyone who needs to avoid alcohol completely for health or ideological reasons. Though 5\%-10\% of users report transient 'healing reactions' in which negative emotions become more obvious, or there are physical symptoms suggestive of toxins being cleared from the system.

\section{REFERENCES}

1. Bach Edward.(1936) the twelve healers and other remedies, London: C.W.Daniel.

2. Bach, E. 1977a. "Heal Thyself." In The Bach Flower Remedies. New Canaan, Conn.: Kears. Originally published by C.W. Daniel, Essex, England, 1931.

3. Chancellor, P. M. 1971, Handbook of the Bach Flower Remedies. London: C. W. Daniel.

4. Christine Zaza and Natalie Baine(2002) Cancer Pain and Psychosocial Factors:Journal of Pain and Symptom Management Volume 24, Issue 5 , Pages 526-542.

5. Cornelia Richardson-Boedler(2007)Applying Homeopathy Bach flower Therapy to Psychosomatic Illness, B jain publishesrs ,New delhi.

6. Cram JR, Kasman G and Holtz J. Introduction to Surface Electromyography. Aspen Press, Gaithersberg, MD. 1997

7. David Lord(1999) Bach Flower Remedies: Diagnosis and Treatment, Astrolog Publishing House,

8. Element Books, Incorporated(1999) Stress Buster: Aromatherapy Massage Bach Flower Remedies, Element Books Limited. 
9. Estella Ritter(2007) The Treatment of Anxiety \& Panic With Bach Flower Remedies: God's Own Medicine, Xlibris Corporation.

10. Jane Evans(1974) Introduction to the Benefits of the Bach Flower Remedies, Ebury Publishing.

11. Jennifer Barraclough(2012) Bach flowers for mind - body healing, Published by Jennifer Barraclough at Smashwords.

12. Judy horward(2007) Do Bach flower remedies have a role to play in pain control?, Complementary Therapies in Clinical Practice,Volume 13, Issue 3 , Pages 174-183

13. Kaslof, L. J. 1988. The Bach Remedies: A Self-help Guide. New Canaan, Conn.: Kears

14. Kylie Thaler, Angela Kaminski, Andrea Chapman, Tessa Langley and Gerald Gartlehner(2009) Bach Flower Remedies for psychological problems and pain: a systematic review, BMC Complementary and Alternative Medicine, 9:16

15. McCutcheon, Lyn.,(1995) "Bach Flower Remedies: Time to Stop Smelling the Flowers.", Skeptical Inquirer, vol. 19, \#4, pp.33-35,55.

16. Robert Halberstein, Lydia DeSantis, Alicia Sirkin, Vivian Padron-Fajardo, Maria OjedaVaz(2007) Healing With Bach® Flower Essences: Testing a Complementary Therapy, Journal of Evidence-Based Complementary \& Alternative Medicine January, vol. 12 no. $13-14$.

17. Scott Kessman(2005) Bach Flower Remedies for Relieving Stress and Other Negative Emotions in People and Animals, http://voices.yahoo.com

18. Tyler, V. E. 1993. "Paraherbalism Is a Pseudoscience." In The Health Robbers, ed. by S. Barret and W.T. Jarvis. Buffalo, N.Y.: Prometheus.

19. Weeks, N. (1973), The Medical Discoveries of Edward Bach, Physician. New Canaan, Conn.: Kears. Originally published in 1940. Weisglas, M. S. 1979. "Personal growth and conscious evolutionn through Bach Flower Essences." Dissertation Abstracts International, part B, p. 3614 (1981).

20. Wheeler, E. J. 1977, "The Bach Remedies Repertory." In The Bach Flower Remedies. New Canaan, Conn.: Kears. Originally published by C. W. Daniel, London, 1952.

21. Wigmore, 1993. Bach Flower Essences for the Family. London: published by author.

22. Vinton McCabe (2009) The Healing Bouquet: Exploring Bach Flower Remedies, ReadHowYouWant.com, Limited 